\title{
Evaluation of the Direct Health Facility Financing Program in Improving Maternal Health Services in Pangani District, Tanzania
}

\author{
Samwel Marco Tukay $\mathbb{D}^{1-3}$ \\ Liliane Pasape \\ Kassimu Tani ${ }^{3}$ \\ Fatuma Manzi ${ }^{3}$ \\ 'The Nelson Mandela African Institution \\ of Science and Technology, Arusha, \\ Tanzania; ${ }^{2}$ Pangani District Council, \\ Tanga, Tanzania; ${ }^{3}$ Ifakara Health Institute \\ (IHI), Dar es Salaam, Tanzania
}

Background: Maternal morbidity and mortality remain significant public health concerns globally, with Tanzania reporting 398 deaths per 100,000 live births annually. While national level data provide some insights into the issue, a focus on sub-national levels is required because of differences in contexts such as rural-urban disparities in maternal mortality. This study examined Direct Health Facility Financing (DHFF) and its effects on the quality of maternal health services in Pangani, a rural district in Tanzania.

Methods: This study was conducted in Pangani district of Tanga region in Tanzania. The study used both qualitative and quantitative methods, including 16 in-depth interviews with the council health management teams, facility in charges, maternity nurse in charge, and 5 focus group discussions with community health governing committee members. The number of deliveries that occurred in health facilities, as well as medical supplies, equipment, and reagents purchased by the facilities, were compared using descriptive statistics before and after the DHFF implementation.

Results: Direct disbursement of funds from the central government through the Ministry of Finance and Planning to the primary health facilities reduced delays in procurement, improved community outreach services, and improved community leaders' engagements. Deliveries occurring at health facilities increased by $33.6 \%(\mathrm{p}<0.001)$ one year after the HDFF implementation. Various medicines, delivery kits, and some reagents increased significantly $(p<0.05)$. However, the lack of computers and poor internet connectivity, an insufficient supply of medical equipment and unstable stock of the Medical Stores Department increased the difficulty of obtaining the missed items from the selected prime vendor.

Conclusion: Overall, this study shows a positive impact of the DHFF on maternal health service delivery in Pangani district. Specifically, an increase in the number of medical supplies, equipment, and reagents necessary to provide maternal health services contributed to the observed increase in facility deliveries by $33.6 \%$. Moreover, the system minimizes unnecessary delays in the procurement processes of required drugs, supplies, and other facility reagents. To maximize the impact of the HDFF system, lack of computers, unstable internet, limited knowledge of the staff about the system, and inadequate health workforce should be addressed. Therefore, strengthening the DHFF system and staff training in-service and on the job is essential for smooth implementation.

Keywords: health financing, direct health facility financing, health governing committee, maternal health, quality of care, Tanzania

\section{Background}

Maternal mortality and morbidity are significant public health concerns globally, with 830 women dying from pregnancy- and childbirth-related complications 
every day. ${ }^{1-4}$ In low and middle-income countries, maternal mortality is high. ${ }^{2,5}$ Tanzania for example, records 398 deaths per 100,000 live births. ${ }^{2}$ The primary causes are maternal haemorrhage, sepsis, hypertensive disorders during pregnancy, obstructed labor, abortion, direct and indirect maternal disorders, Human Immunodeficiency Virus (HIV), and other pregnancy-related infections. ${ }^{2,6,7}$ Provision of quality health services during prepregnancy, pregnancy, delivery, and postnatal is critical for survival. ${ }^{8,9}$ Specifically quality maternal health care is vital for preventing and reducing the causes of obstetric complications, stillbirths, maternal and newborn deaths. ${ }^{9}$ However, like other developing countries, Tanzania's health-care systems face challenges such as lack of adequate medical equipment or outdated equipment and insufficient health facility staff. ${ }^{10-14}$ Other challenges include limited availability of drugs and medical supplies, limited access to medical aid and insurance schemes, referral systems, and a culture of informal payments. ${ }^{15}$ In addition, rural-urban disparities in maternal mortality ratio exist across various regions of Tanzania, with urban health facilities being of higher quality than their rural counterparts. ${ }^{16}$ To improve maternal health services in rural communities, establishing financing protection mechanisms by imposing mandatory regulations is critical. ${ }^{17,18}$ Health systems financing determines the quality of health care available. ${ }^{19}$ Health financing also influences the quality of care by coordinating policy and implementating of proper health system functions. ${ }^{20}$ This is in line with accessing maternal health care as one of the aspects of attaining universal health coverage (UHC) that affects women, their families, communities, and nations at large. ${ }^{21}$ Since independence in 1961, the government of Tanzania has aimed at providing quality and affordable health-care services from tax resources and user fees. ${ }^{22}$ Various health financing reforms have been introduced to tackle maternal and other health-related problems. ${ }^{23}$ The main objectives of those reforms are: to raise sufficient resources for health, remove financial risks and barriers to access, eliminate resource waste and overall promote efficiency, improve the quality of health care, reduce high out of pocket expenditure, increase the utilization of healthcare services and improve health outcomes. ${ }^{24,25}$ Previous reforms initiatives like the 1999 Decentralization by Devolution, saw funds disbursed to District Medical Officers (DMOs) rather than officers in charge of health facilities. ${ }^{26,27}$ In practice, this resulted in delays in fund disbursements from DMOs to primary health-care facilities and conflicts between DMOs and officers in charge of the health facilities. ${ }^{26,28}$ In 2011, Tanzania launched the Pay for performance (P4P) program to strengthen health services delivery in terms of availability, accessibility, and acceptability. ${ }^{29,30}$ There has been a marked increase in the availability of maternal health drugs provided free of charge and increased financial incentives for health-care workers, resulting in improvements in inpatient care. ${ }^{30}$ Health-care providers and facilities were awarded financial incentives based on achieving nine maternal and child health goals. ${ }^{31}$ Health-care providers who achieved $75 \%$ of the goals were given full payment and $50 \%$ to those who did not reach the goals. ${ }^{31}$ However, it was portrayed that, during the implementation of P4P, Reproductive and Child Health (RCH) staff received better financial rewards than their non-RCH counterparts. ${ }^{32,33}$ However, results showed that the costs of managing the P4P program were higher than the costs of actual incentive payment. ${ }^{31,34}$ Further, in health facilities with limited staff, the tasks associated with managing the P4P data negatively impacted the other health services delivered. ${ }^{31,34}$

The issue of primary health-care facilities financing in most African countries is at a crossroads as resources for health are limited, and the elimination of user fee policies has been poorly implemented with no alternative sources of facility income. ${ }^{35}$ When implemented effectively however, health financing initiatives results in tangible outcome because health systems provide quality services and are more responsive to clients. ${ }^{36}$ Healthcare facilities' governance is a partnership between facility staff and community representatives, with health systems being more responsive and people-centred. ${ }^{45,46}$ In countries like Congo, the government subsidized the cost of accessing medical services and introduced a health financing system that played a significant role in eliminating maternity and delivery-related fees and consequently reduced maternal and child mortality rates. ${ }^{14,39,40}$

In 2018, Tanzania introduced fiscal decentralization, namely, Direct Health Facility Financing (DHFF) to primary health facilities. The goal was to grant planning, management, and financial autonomy to primary healthcare providers. ${ }^{36,41}$ Furthermore, the DHFF was expected to increase health system transparency, accountability and community participation. ${ }^{41}$ The health basket funds are released from the central government level and allocated to local government authorities quarterly. ${ }^{42,43}$ Experiences 
of the DHFF implementation showed positive outcomes as health workers could better plan and budget for their activities. ${ }^{44}$ Also, this initiative improved governance and accountability and led to reduced stress among health staff. $^{41,43,44}$ Effectively implemented health financing leads to health systems that are responsive to beneficiaries and provides quality services. ${ }^{36}$ Where governance of health-care facilities is a partnership between facility staff and community representatives, health systems are responsive and people-centred. ${ }^{37,38}$ This study used a mixed-methods approach to assess early effects of the DHFF programme implemented by the government of Tanzania on maternal health in Pangani district.

\section{Methods}

\section{Research Setting}

This study was conducted in Pangani district, geographically located in northeast Tanzania. The district is generally rural, and according to the 2012 Tanzania Population and Housing Census, it had a population of 54,025 people. The female population numbers 27,155 , which is $50.27 \%$ of the total population, with an average household size of approximately 4 persons. The population density was 24 persons per square kilometre, with about $21 \%$ of the population living in a semi-urban centre. The main socialeconomic activities are cash crops, food crops, livestock keeping, poultry keeping, fishing, and small-scale industries.

In February 2018, Tanzania officially launched the DHFF system policy. ${ }^{47}$ This policy aims to devolve planning, budgeting, and implementation responsibilities to primary health providers.

The program was introduced to meet the following goals: improving the structural quality of maternal and child health services, increasing accountability and governance in the health system at the primary health-care facilities, facilitating increasing health system responsiveness to patients who receive health-care in the respective health facilities, and improving health-seeking behaviour and service utilisation at the primary health facility level. ${ }^{41,48}$

This is a marked departure from the previous system where planning and budgeting were done by DMOs. Another aim of the DHFF is to improve the sense of community ownership of health-care provision by including community representatives in health facility governing committees. $^{47,49}$ Under the DHFF program, the Council
Health Service Boards and health facility governing committees have been given the primary responsibility to undertake planning, budgeting, and implementing health service delivery at the local level. ${ }^{47,49}$

Before the DHFF, disbursement of funds to Primary Health facilities passed through 2 levels; from the central government through the Ministry of Finance and Planning to the President's Office-Regional Administration and Local Government and then the respective Local Government Authorities. ${ }^{41,50}$

Under local government authorities, the DMOs received the health budgeted funds, and disbursed the funds to primary health-care facilities. ${ }^{51}$ Delays were sometimes encountered in disbursements of the funds by DMOs, which negatively impacted the delivery of health services, including those for vulnerable women and led to negatively impacted service delivery by consumers. ${ }^{47,52}$ The DHFF system acts as a bridge between central government (Ministry of Finance and Planning) and local government authorities the primary health facilities in which funds are directly transferred from the Ministry of Finance and planning to frontline primary health facilities. $^{53}$ The shift aimed to increase service delivery efficiency by reducing leakages and enhancing predictability and timeliness of funds received at primary health facilities. $^{41,54}$

PlanRep is a planning and reporting database used by local government authorities during planning and reporting, facility budget is governed by previous financial year annual revenue collection as budget sealing cut off point from central government to local government and partly is driven by consideration of population size of respective health facilities. ${ }^{53,54}$

In the four-year lifespan of the DHFF in Pangani, there has not been a thorough assessment of its impact on maternal health services. ${ }^{47}$ This study attempts to make such an evaluation, and we consider that the implementation of the DHFF is timely to improve the quality of and access to maternal health services.

Under the DHFF, the facility therapeutic committee must consider previous stock and available funds when planning the procurement of maternal drugs such as folic acids, iron supplements, magnesium sulphate, and sulfadoxinepyrimethamine (SP) for malaria treatment; medical reagents such as syphilis test, malaria rapid diagnostic test, and deep urine stick for protein; medical equipment like blood pressure machine, weighing scale machine, fetal scope, and delivery kit. The facility pharmacist prepares the Medical 


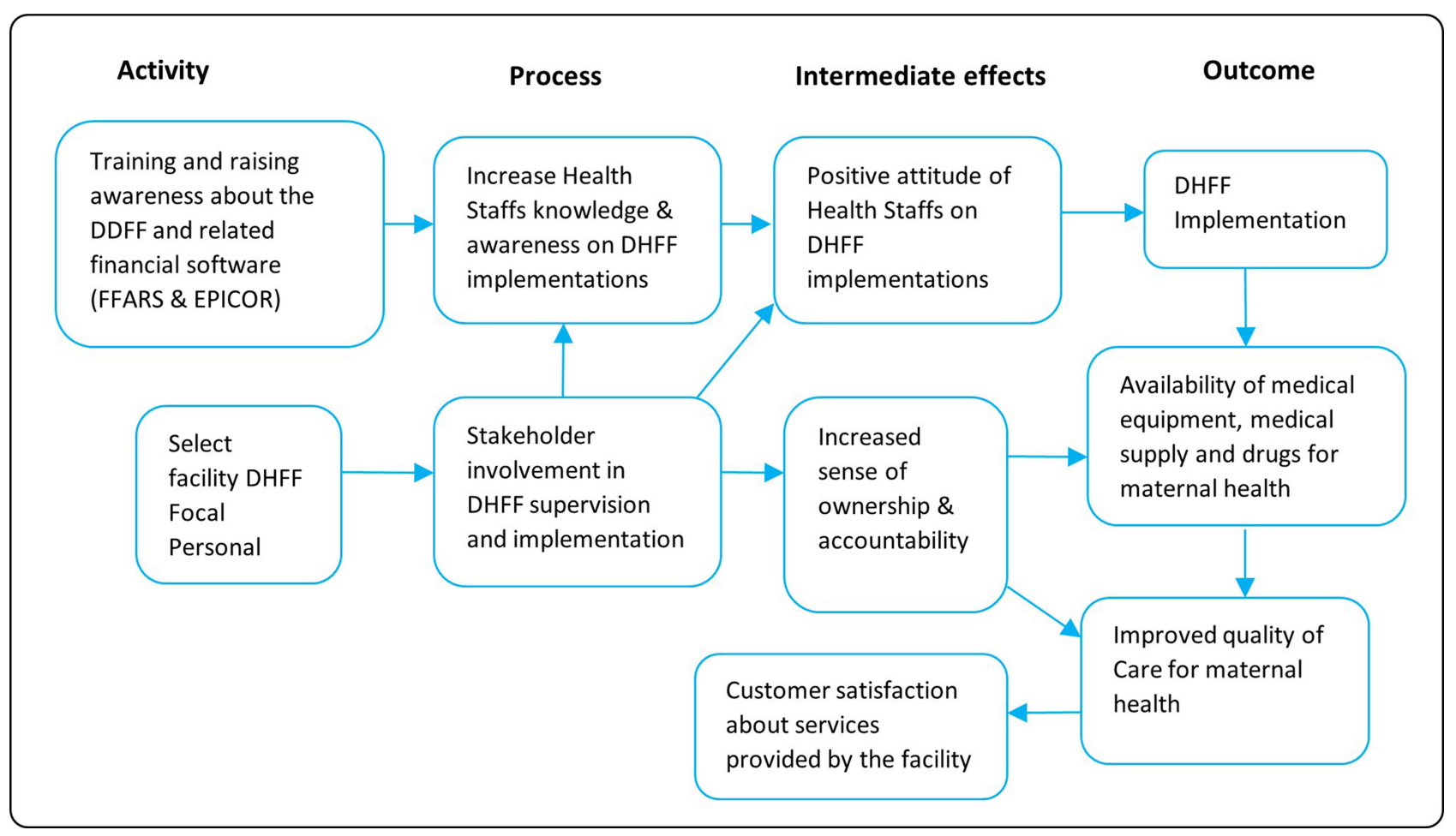

Figure I Theoretical framework for DHFF implementation in the study area.

Stores Department (MSD) orders through an electronic logistic management information system. Once approved by the medical officer in charge of the facility, the order is sent to MSD. MSD prepares a pro forma invoice for available and out-of-stock items, which are then sent to the facility for payment of available items. Out-of-stock items are sourced from approved private vendors by the procurement management unit, which issues a local purchase order to the vendor. ${ }^{55}$ Once the facility therapeutic committee and community health governing committees approve items delivered by the vendor, payment is then made.

\section{Study Design}

This study used a mixed-methods approach, utilizing both qualitative and quantitative data. The qualitative data were based on interviews and focus groups, hence consistent with the Consolidated Criteria for Reporting Qualitative Research (COREQ) ${ }^{56}$ The quantitative data compared births occurred at health facilities before and after the DHFF intervention. The study the used financial year 2016/2017 as a source for baseline data for the period before introducing DHFF and the financial year 2017/ 2018 as the follow-up year after the intervention.

To evaluate outcome on facility deliveries, availability of essential drugs, medical equipment, and reagents for maternal health and quality of care provided for maternal health, fidelity of implementation framework ${ }^{57-59}$ was used. The study was implemented based on the structured conceptual framework as shown in Figure 1. The framework was adapted from Ntuli's ${ }^{47}$ to enable the establishment of the effect pathway relationship to determine the trend and progress of DHFF interventions.

\section{Sample Size}

The study used a purposive sampling approach with stratification to select participants from five ${ }^{5}$ health facilities, namely, Pangani district hospital, Mwera health center, and three dispensaries: Masaika, Kwakibuyu, and Mkalamo. A reason for choosing these facilities was to have a representative sample of health facilities across different levels of service provision at the district level: hospital, health center, and dispensary. Also, the study selected these facilities because they had a high volume of deliveries compared with others of the same level within the district. Therefore, the choice of these facilities enabled us to access a large quantity of information related to our study objectives. The number of deliveries from the facilities before HDFF (2016/17) and one year thereafter (ie, 2017/18) was 1189 and 1589, 
Table I Status of Facility Deliveries Before and After the DHFF Implementation in Pangani District

\begin{tabular}{|c|c|c|c|c|}
\hline & $\begin{array}{r}\text { Number of Deliveries } \\
\text { Before the DHFF } \\
\text { Implementation }\end{array}$ & $\begin{array}{r}\text { Number of Deliveries I } \\
\text { Year Following the DHFF } \\
\text { Implementation }\end{array}$ & $\begin{array}{r}\text { Change in the } \\
\text { Number of } \\
\text { Deliveries }\end{array}$ & \\
\hline Facility Name & $A(2016 / 17)$ & B $(2017 / 18)$ & B - A & P-value \\
\hline District Hospital & 875 & 1108 & $233(26.6 \%)$ & $<0.001$ \\
\hline Mwera Health Center & 124 & 240 & 116 (93.5\%) & $<0.001$ \\
\hline Masaika Dispensary & 38 & 42 & $4(10.5 \%)$ & 0.045 \\
\hline Kwakibuyu Dispensary & 40 & 62 & $22(55.0 \%)$ & $<0.001$ \\
\hline Mkalamo Dispensary & 112 & 137 & $25(22.3 \%)$ & $<0.001$ \\
\hline All facilities (total) & 1189 & 1589 & 400 (33.6\%) & $<0.001$ \\
\hline
\end{tabular}

Notes: "A" represents financial year 2016/17 and "B" represents financial year 2017/I8. P-value $<0.05$ indicates that the difference is statistically significant.

respectively (Table 1). Number of units of different medical supplies, equipment and reagents are shown in Table 2.

\section{Data Source}

Data collection procedures for both quantitative and qualitative data involved visiting each study facility and meeting all key participants and key informants to capture information relevant to the study objectives. This was consistent with the principles for process evaluation and mixed methods research. ${ }^{60}$ Quantitative data were collected to assess changes over time from facility registry for 2016/2017 as a baseline year before the introduction of DHFF and 2017/2018 as follow-up year after the DHFF intervention was implemented. Health-care workers and health governing committee members from each study facility were interviewed based on their experiences before and after introduction of DHFF to describe changes over time. The qualitative data was collected by interviewing the key informants, through in-depth interviews and focus group discussions (FGDs). The reason why we have conducted qualitative part of our study is to explore the reality of DHFF system implementation success and challenges direct from primary health facilities. During the development of the questionnaire and interview guide, different steps were taken as stated below:

(i) Reviewing the hypothesis and clear understanding of the objectives of the study.

(ii) Assessing the relevance of the methods in relation to the problem

(iii) Nature of the data needed. (iv) Identification of appropriate respondents.

(v) Development of data collection tools. At this step, we also considered the use of language that was familiar to the respondent, the use of simple words that held the same meaning as the respondent's, avoiding jargon, and minimizing long questions.

(vi) Determine the relationship of the questions according to the different subtopics

(vii) Prepare the interview guide/questionnaire for pretesting.

Participants were informed and consented to the publication of anonymised responses.

Data collection tools were piloted for five days by the research team in Bagamoyo district. The district was selected for the pilot survey because it is similar in characteristics with Pangani district where the actual study was conducted. All challenges and observations noted during the pilot survey were addressed before the actual field data collection.

Qualitative data analysis was undertaken using a framework grouping of relevant themes that answers key issues as per study objectives. Transcripts were managed and coded in NVIVO 11 by the use of the framework analysis approach. ${ }^{61}$ Coded data were reviewed, organized into charts according to research themes focusing on the study objectives and emerging issues from the interviews.

\section{Data Validation}

After development of the questionnaire and interview guide, different purposive selections of respondents were 
Table 2 Status of Medical Supplies, Medical Equipment, and Medical Reagents Before and After the DHFF Implementation in Pangani District

\begin{tabular}{|c|c|c|c|c|c|}
\hline & & $\begin{array}{r}\text { Number Before } \\
\text { the DHFF } \\
\text { Implementation }\end{array}$ & $\begin{array}{r}\text { Number I Year } \\
\text { Following the } \\
\text { DHFF } \\
\text { Implementation }\end{array}$ & Change & \\
\hline Variable & $\begin{array}{l}\text { Unit of } \\
\text { Measurement }\end{array}$ & $A(2016 / 17)$ & B $(2017 / 18)$ & B - A & P-value \\
\hline \multicolumn{6}{|l|}{ MEDICINE } \\
\hline Folic Acids & $5 \mathrm{mg}$ Tabs & 3200 & 12,000 & $8800(275.0 \%)$ & $<0.001$ \\
\hline Iron supplement & $\begin{array}{l}\text { (Ferrous Sulphate } \\
200 \mathrm{mg}+\text { folic Acid } \\
0.25 \mathrm{mg} \text { ) Tabs }\end{array}$ & 18,450 & 19,950 & $1500(8.1 \%)$ & $<0.001$ \\
\hline Magnesium Sulphate & $50 \%$ W/V Injection & 70 & 95 & 24.8 (35.3\%) & $<0.001$ \\
\hline Sulfadoxine-pyrimethamine & (Tabs) & 6020 & 6160 & 140 (2.3\%) & $<0.001$ \\
\hline \multicolumn{6}{|l|}{ MEDICAL EQUIPMENT } \\
\hline Blood Pressure Machine & Piece & 2 & 8 & $5.8(241.7 \%)$ & 0.070 \\
\hline Weighing Scale Machine & Piece & 2 & 4 & $1.4(58.3 \%)$ & 0.322 \\
\hline Fetal Scope & Piece & 3 & 4 & $1.4(53.8 \%)$ & 0.252 \\
\hline Delivery Kit & Packs & 265 & 513 & $248.2(94.8 \%)$ & $<0.001$ \\
\hline \multicolumn{6}{|l|}{ MEDICAL REAGENTS } \\
\hline Syphilis Test (RPR) & Strips & 2348 & 6142 & $3794.5(161.6 \%)$ & $<0.001$ \\
\hline Malaria Rapid Diagnostic Test & Strips & 4185 & 9425 & $5240(125.2 \%)$ & $<0.001$ \\
\hline Urine Deep Stick for Protein & Strips & 3140 & 1800 & $-1340(-42.7 \%)$ & $<0.001$ \\
\hline
\end{tabular}

Notes: "A" represents financial year 2016/17 and "B" represents financial year 2017/18. P-value $<0.05$ is statistically significant.

selected in different sites. After obtaining their consent, we conducted interview using the entire respective questionnaire and interview guide. During this pilot/pre-testing we identified questions that needed to be adjusted and/or improved.

\section{Quantitative Data}

At each facility, the study extracted retrospective information from the health facilities' information system books: book number 4 - ledger book, book number 6 - antenatal care register, book number 12 - labors, and delivery book, and book number 13 - postnatal register. Data was also extracted from the facility Health Management Information System registry. The variables recorded were services provided, medical equipment, maternal drugs, and maternal medical reagents.

\section{Qualitative Data}

This study conducted 16 In-depth Interviews (IDIs) with health-care workers and 5 Focus Group Discussions (FGDs), which comprised 8-12 participants. The participants were community health governing committee members from each facility. The principal investigator (ie, Samwel Tukay) used a guiding tool during the collection of qualitative data. There was no prior relationship between the participants and the research team. The investigator took notes during IDIs, and FGDs sessions and all sessions were audio-recorded to facilitate transcription of the information. Digital recordings of interviews were made and transcribed in Kiswahili and then translated to English. The study used digital voice recorder, pen, pencils, and notebooks to capture participants' information and critical issues to answer research objectives. All the 
audio interviews were transported from the audio recorders to the laptop for further processing. All the interviews were transcribed word for word by the researcher. After transcription, all the transcripts were cross-checked in relation to the audio recording to ensure all the information have been captured correctly as it is in the audio without omission of any information. All the correct transcripts were then translated from Swahili to English by an experienced translator, and all the translated transcripts were also checked for clarity before analysis.

\section{Data Analysis}

The quantitative data analysis was done descriptively using Stata (version 12) statistical software. The number of deliveries and those of different medical supplies, equipment, and reagents were compared before and after DHFF implementation using a two-sample test of proportions.

Qualitative data analysis was undertaken using framework analysis. We grouped relevant themes that answer key issues as per study objectives. Coded data were reviewed, organized into tables according to research themes focusing on the study objectives and issues emerging from the interviews. Further details about the coding are presented in the data analysis by framework grouping (Table 3).

\section{Results}

\section{Stakeholders' Involvement}

Key stakeholders involved in the implementation of DHFF are health-care workers, facility accountants, and community representatives sitting as health facility governing committee members. Each has a different role in the management of funds disbursed to health facilities. A medical officer in charge of the health facility and health governing facilities are responsible for authorizing facility transactions supported by facility health accountants. The accountant ensures that the financial management of facility accounts is in line with DHFF standards and guidelines.

“... According to DHFF guidelines, health governing committee members are the ones responsible for the authorization of any facility transaction, and the facility incharge and the facility accountant are skilled personnel in finance responsible for all matters related to the facility accounts ..." Community health governing committee members, FGDs
Interviews with these stakeholders revealed that before DHFF, the funds and all requirements were requested by the Council Health Management Team (CHMT). Many times, the CHMT planned things that were not priorities at the facility and neglected the actual demand of the health facility. It took a long time to receive money, and the amount was not sufficient, which compromised the implementation of activities.

"... With the previous approach, CHMT was given the mandate to plan on behalf of the facility, and many times they planned things which are not priorities at the facility and neglect the real demand of our health facility ..." (Community health governing committee member, FGDs)

“ ... previous system stock of drugs from MSD was issued on a quarterly basis in a small amount which was not satisfying the facility needs and all requests from the facilities were submitted to DMO office for approval. This was financially expensive and created some delay ... ". (CHMT Level Participant, IDI)

The introduction of the DHFF was reported to open more stakeholders' involvement as the facilities started to directly manage their own funds. This made it easier for health facilities to plan on their own, based on their priorities. Each facility in charge and maternity nurse in charge are responsible for counterchecking their facility budget to see if their budget considers maternal and child health. In this new approach, the time between request submission and receipt of the funds had been notably reduced.

“... With this new system, health facility can procure their own needs at the right time and reduce unnecessary delays in health services provision as compared to the previous approach ...". (CHMT Level Participant, IDI)

With this new DHFF system, all health facility staff and community health governing committees are involved in all matters related to the health facility, including budget preparation and review of expenditures for maternal health services. Health governing committee members were reported to be the primary owner of the health facility. It was said that no activity could be done without their permission. For health-care workers, they are directly involved at all levels of planning and decisions making, because they are the ones who identify needs in their areas and plan what to do. The stakeholders were quoted as saying: 
Table 3 Data Analysis by Framework Grouping

\begin{tabular}{|c|c|c|c|}
\hline Themes & Definition & $\begin{array}{l}\text { Codes } \\
\text { and } \\
\text { Sub } \\
\text { Codes }\end{array}$ & Example \\
\hline \multicolumn{4}{|c|}{ Objective I: The direct health facility financing implementation success. } \\
\hline $\begin{array}{l}\text { Knowledge prior and } \\
\text { post DHFF interventions }\end{array}$ & $\begin{array}{l}\text { On this section, we wanted to know how facility } \\
\text { staffs and community health governing committee is } \\
\text { aware about DHFF. }\end{array}$ & $\begin{array}{l}\mathrm{CHMT} \\
\mathrm{CHGCM} \\
\mathrm{HFI}\end{array}$ & $\begin{array}{l}\text { This is a new health financing system introduced by } \\
\text { the government where facility fund is direct } \\
\text { channelled to facility bank account and facility } \\
\text { health governing committee members in } \\
\text { collaboration with health-care providers are } \\
\text { planned on how to use it (PI, CHMT, IDIs). }\end{array}$ \\
\hline Reflections on training & $\begin{array}{l}\text { On this section, we wanted to know how if } \\
\text { participants manage to mention factors which will } \\
\text { helps to strengthening DHFF system }\end{array}$ & $\begin{array}{l}\mathrm{CHMT} \\
\mathrm{CHGCM} \\
\mathrm{HFI}\end{array}$ & $\begin{array}{l}\text { As facility health governing committee members, } \\
\text { we do not have enough knowledge on how this } \\
\text { new system is working (CHGCM, FGDs). }\end{array}$ \\
\hline Finance. & $\begin{array}{l}\text { On this section, we would like participants to } \\
\text { mention challenges which contribute in DHFF } \\
\text { system success implementations }\end{array}$ & $\begin{array}{l}\text { CHGCM } \\
\mathrm{HFI}\end{array}$ & $\begin{array}{l}\text { A delay in fund disbursement from the government } \\
\text { to the facilities led to underperformance. (HFI (4), } \\
\text { IDIs). } \\
\text { All facility funds are controlled by facility in charge } \\
\text { and facility health accountants. We facility health } \\
\text { governing committee members our responsibility is } \\
\text { to overseen and countercheck formal financial } \\
\text { procedures. (CHGCM (9), FGDs). }\end{array}$ \\
\hline Involvement & $\begin{array}{l}\text { On this section, we would like to know if } \\
\text { community health governing committee members } \\
\text { they are involved in planning, budgeting and } \\
\text { purchasing of drugs, medical supplies and medical } \\
\text { reagents }\end{array}$ & CHGCM & $\begin{array}{l}\text { According to DHFF guidelines, community health } \\
\text { governing committee members are the ones } \\
\text { responsible for the authorization of any facility } \\
\text { transaction, and the facility in-charge and the facility } \\
\text { accountant are skilled personnel in finance } \\
\text { (CHGCM (2), FGDs). } \\
\text { Normally in our facility team comprise a mixtures } \\
\text { of medical personnel who is understanding all } \\
\text { essential requirement for maternal health when } \\
\text { preparation of facility action plan is done they do } \\
\text { plan on behalf of these committee members but } \\
\text { committees they must know each steps and what } \\
\text { was planned so that we can give approval (CHGCM } \\
\text { (5), FGDs). }\end{array}$ \\
\hline \multicolumn{4}{|c|}{$\begin{array}{l}\text { Objective 2: To assess effect of DHFF on quality of care for maternal health-care in terms of availability of essential medicine, } \\
\text { supplies and medical equipment. }\end{array}$} \\
\hline Quality of care & $\begin{array}{l}\text { On this section, we would like participants to } \\
\text { mention the outcome on quality of care provided } \\
\text { for maternal health after introduction of DHFF }\end{array}$ & $\begin{array}{l}\text { CHGCM } \\
\mathrm{HFI}\end{array}$ & $\begin{array}{l}\text { This new health financing system is good compared } \\
\text { to previous approach because with previous system } \\
\text { stock of drugs from MSD was issued on quarterly } \\
\text { bases in small amount which do not satisfy facilities } \\
\text { demand (CHGCM (5), FGDs). } \\
\text { DHFF intervention enabled health facility to own } \\
\text { their own fund when they face shortage of essential } \\
\text { medicine, supplies and medical equipment for } \\
\text { maternal health they can raise request and make } \\
\text { ordering at appropriate time (HFI (3), IDI). }\end{array}$ \\
\hline
\end{tabular}

(Continued) 
Table 3 (Continued).

\begin{tabular}{|c|c|c|c|}
\hline Themes & Definition & $\begin{array}{l}\text { Codes } \\
\text { and } \\
\text { Sub } \\
\text { Codes }\end{array}$ & Example \\
\hline $\begin{array}{l}\text { Availability of delivery } \\
\text { materials }\end{array}$ & $\begin{array}{l}\text { On this section, we wanted to know the availability } \\
\text { of essential delivery equipment's. }\end{array}$ & $\begin{array}{l}\text { CHMT } \\
\mathrm{HFI}\end{array}$ & $\begin{array}{l}\text { The DHFF helps our dispensary to procure } \\
\text { requirements needed for reproductive health both } \\
\text { within dispensaries and or at community level } \\
\text { during outreach and follow up services by doing so } \\
\text { helps our dispensary to stand alone in planning, } \\
\text { purchasing of drugs necessary for maternal health } \\
\text { like delivery packs, folic acid, and other maternal } \\
\text { health related requirements (HFI (I), IDls). }\end{array}$ \\
\hline \multicolumn{4}{|c|}{ Objective 3: To evaluate the outcome of DHFF on quality of care provided for maternal health } \\
\hline $\begin{array}{l}\text { Improved and quality } \\
\text { maternal health services } \\
\text { at primary health } \\
\text { facilities. }\end{array}$ & $\begin{array}{l}\text { On this section, we wanted to know positive effect } \\
\text { of introduction of DHFF at primary health facilities. }\end{array}$ & $\begin{array}{l}\text { CHMT } \\
\mathrm{HFI}\end{array}$ & $\begin{array}{l}\text { With previous approach we were not involved in } \\
\text { any facility decision making. But with this new } \\
\text { system there is a great change in which facility } \\
\text { health governing committee members are fully } \\
\text { involved in all matters related to our health facility } \\
\text { by doing so increase sense of ownership and trust } \\
\text { to the community (CHGCM (4), FGDs). }\end{array}$ \\
\hline \multicolumn{4}{|c|}{ Objective 4: To explore challenges facing direct health facility financing in provision of maternal health. } \\
\hline $\begin{array}{l}\text { DHFF system } \\
\text { operational obstacles }\end{array}$ & $\begin{array}{l}\text { On this section, we wanted to understand } \\
\text { challenges facing health-care workers and the } \\
\text { community health governing committee members } \\
\text { in the system implementation process. }\end{array}$ & $\begin{array}{l}\mathrm{HFI} \\
\mathrm{CHGCM} \\
\text { CHMT }\end{array}$ & $\begin{array}{l}\text { Delay in release of other charges (Oc) funds cause } \\
\text { lot of complications as we have limited celling in our } \\
\text { basket fund budget the only source we are } \\
\text { depending on is other charges (CHMT (2), IDIs). } \\
\text { We do not have enough education on how this } \\
\text { system is working (CHGCM (I), FGDs). }\end{array}$ \\
\hline
\end{tabular}

“... Members of the governing committee were involved in all stages from planning and implementation of the plans, and at all times the health facility administration needed to do their activity during planning; budgeting and purchasing of drugs, medical supplies, and medical reagents ...". (CHMT Level Participant, IDI)

CHAIRPERSON" ... I am a chairman for all matters related to this facility; therefore, no plans or implementation can be done in the facility without my involvement. By so doing, I am fully involved ..." (Community health governing committee members, FGDs)

In general, most of the included stakeholders were those who knew the essential requirements for maternal health. Some had worked in the facility for two or more years, and others attended different DHFF capacity-building training sessions. The health governing committee members, as community representatives participated in all stages in the planning, budgeting, and implementation, including purchasing of drugs, medical supplies, and medical reagents. The committee members were involved in authorization, and when drugs were received, they were involved in verification. Again, they were involved at all times they were needed, like decision-making during challenges about drug deficit or any other issue related to the facility.

\section{Operational Successes and Challenges}

The DHFF helped the facility to provide better maternal health services. As funds were directly allocated to the facility bank account, it helped timely purchase of maternal health requirements. It was quoted as follows:-

“... More funds were released to our health facility and helped to purchase modern equipment for maternal health ..." (Facility in charge, IDI)

On-the-job training was conducted for health-care workers and health governing committee members as part of the 
DHFF system, which helped provide good maternal health care.

Health workers reported that it helped them procure various reproductive health requirements needed for care delivery at the facility and outreach services in the community. It gave the mandate to facility staff to set plans and priorities basing on facility needs; it reduced the time from ordering and receiving facility items. It included planning timely procurement of needed supplies and making decisions based on needs. Health workers were quoted as saying:

“... helps our dispensary to procure requirements needed for reproductive health both within dispensaries and or at community level during outreach and follow up services by doing so helps our dispensary to stand alone in planning, purchasing of drugs necessary for maternal health like delivery packs, folic acid, and other maternal health requirements ..." (Facility in charge, IDI)

“... DHFF provides a chance for health facility staffs to make a decision basing on their needs and priorities for mother and child health as this helps to improve maternal health as well as to provide essential requirements for maternal health to the clients at the right time ..." (CHMT Level Participant, IDI)

The early implementation of DHFF was reported to face infrastructural challenges. Some health facilities reported not having computers which is an essential tool during the preparation and initiation of vouchers, raising requests, and storing facility reports. Internet connectivity was poor in this rural district. These challenges may be alleviated by allocating resources to procure and improve the connectivity.

“... We don't have a computer and it's supporting accessories to run this system. This wastes us time and finances to travel to Pangani hospital to produce a voucher, in addition we faced unnecessary delays to process facility requirements due to poor internet connectivity to run this system ..." (Facility in charge, IDI)

“... Sometimes we tried to pay MSD for facility drugs, but we failed due to poor internet connection that led to unnecessary delays to receive drugs from MSD ..." (Facility in charge, IDI)

During IDIs and FGDs with council health management team, health facility in charges and community health governing committee members, they agreed and decided to include purchase of a new computer and strengthening internet connectivity infrastructure in their annual budget plan to overcome this challenge and hence will improve the performance of the DHFF system. This will be done on quarterly basis for two years consecutively to avoid collapsing of other procurement system within the facilities.

Other problems that impacted the smooth provision of maternal health services in DHFF included a shortage of health-care providers, insufficient medical equipment and supplies for maternal health, and also funds directed to the primary facilities were inadequate to meet all needs for maternal health services.

Health workers reported various coping strategies to address DHFF implementation challenges, including traveling long distances to use district headquarters' computers or work late at night to access the internet.

“... I normally go to the DMO office and use their computer to prepare our facility requests, some health facilities they do send facility requests late at night; that is when the internet service is stable ..." (Facility maternity nurse in charge and facility in charge, IDI)

To strengthen the DHFF system so that it functions effectively, interviewees suggested training all staff regarding the system and including it as part of pre-service. It was also advised to install alternative power sources like generators or solar power and recruit enough accountants for each health facility. In conjunction with this it was also proposed that, in the future this system should be part of the syllabus taught in the health institutions, this will help to impart the required skills and knowledge to health-care workers to simplify the implementation of this new system.

“... This system needs to run in parallel with the speed of financial flow in our facility as this will help to implement our planning at the right time and facility staff need to be trained on how to operate this system so that all staff can be aware of how to use this system ..." (CHMT Level Participant, IDI)

“... Every health facility recruits enough number of health accountants to operate this system ..." (Community health governing committee members, FGDs)

As indicated in Table 1, there was a significant increase in the number of deliveries occurring in health facilities, from 1189 before the DHFF to 1589 after one year following the DHFF implementation. This represented a $33.6 \%$ increase in facility deliveries attributable to the DHFF $(\mathrm{p}<$ 0.001). Significant increase in facility deliveries was also 
observed in the individual facilities, ranging from $10.5 \%$ in Masaika Dispensary $(p=0.045)$ to $93.5 \%$ in Mwera Health Centre $(\mathrm{p}<0.001)$.

There were few improvements in the availability of medical equipment. Even though it was documented that customer satisfaction had improved. With increases in essential drugs and equipment, we have witnessed more deliveries at the hospital and health center to which pregnancy mothers were referred from dispensaries. This was supported by qualitative findings that indicate an increase in deliveries in the study district. District hospital and Health center are referral places for lower-level facilities, they receive more cases from lower facilities which need special attention during delivery such as mothers with a history of previous scars, eclampsia, obstructed labor, and prime delivery as per national policy guidelines.

Apart from that, Pangani district is surrounded by the Tanga municipality, Muheza district, and Handeni district. Therefore, pregnant women from neighboring areas benefited from maternal health services from Pangani district and this increased the number of deliveries in this facility.

" ... More than that, community awareness on the importance of health facility delivery is high as compared to the previous situation where majority of pregnant women were attended by traditional birth attendants (TBAs) for delivery. But today TBAs were instructed to be the primary source of referrals for all maternally related cases from the community to health facility for proper delivery care, and they are not allowed to conduct any delivery ..." (CHMT Level Participant, IDI)

Further, the study observed a significant increase in medical reagents and supplies one year after the HDFF implementation. As shown in Table 1, all medical supplies increased significantly, with the lowest but significant increase of $2.3 \%$ for Sulfadoxine-pyrimethamine tablets $(p<0.001)$ and the highest being $275.0 \%$ for folic acid $5 \mathrm{mg}$ tablets $(\mathrm{p}<0.001)$. However, apart from delivery kits which increased significantly by $94.8 \%(\mathrm{p}<0.001)$, there was no evidence of significant increase $(p>0.05)$ in the rest of the medical equipment within the one year of DHFF implementation. There were many reasons for this, including difficulty in procuring items that are missing at MSD and a lack of skilled staff to affect procurement.

There was also a significant increase in medical reagents. Syphilis test strips, and malaria rapid diagnostic test (MRDT) strips increased by $161.6 \%(p<0.001)$, and
$125.2 \%$ ( $\mathrm{p}<0.001)$, respectively. However, there was a significant decline $(\mathrm{p}<0.001)$ in the number of urine deep sticks by $42.7 \%$ (Table 2 ).

During planning and budgeting, facility staff and community health governing committee members prioritize medical supplies, reagents, drugs, and foregone medical equipment. During IDIs and FGDs discussions we noted that: no skilled staff at dispensary level (pharmacist techs, pharmacist assistants, and accountants) to manage facility procurement system, fund management, inadequate money collection from user fees due to a large number of exempted patients (above 65 years, psychiatric patients, under five and chronic illnesses like hypertension and diabetes) this result in low buying power at the facilities.

Items that are not supplied by MSD could be ordered by special procurement at MSD. This process could take up to 6 months or more with no certainty of receiving the required item. The procurement of items not available in the MSD catalogue was made more difficult due to the unavailability of a prime vendor for the Tanga region who would be able to supply items that are unavailable at MSD. More than that, to procure items outside MSD required an out-of-stock confirmation letter from MSD, which is difficult to obtain. Even if one received it, procuring items from local vendors is challenging due to: high prices, inconsistent supply, and difficulty in receiving proper and complete documentation such as Electronic Fiscal Device (EFD) receipts, etc.

“. ... due to unstable stock supply from MSD, health facilities were discouraged by the long wait procurement process resulting in difficulties in procuring medical equipment when funds were available. These funds were then used for other activities ..." . (Facility in charge, IDI)

Health workers and members of the health facility committees felt that the flow of patients for maternal health increased compared to before DHFF. This increase is a result of enough supply of drugs, medical supplies, and medical reagents to facilitate the provision of good maternal health. Health workers said that community outreach services were provided as required because staff could be paid their day-out allowances using the funds available due to the DHFF. They felt that essential requirements for maternal health have improved and that there is high customer satisfaction with maternal health services.

Modern equipment for maternal health are procured as compared to the previous system and that led to an 
increasing number of pregnant women attending our facilities for reproductive health.

Clients are satisfied with health facilities services because most of all essential requirements for maternal health is currently available and the missing gap they use available facility funds to procure. The number of outreach clinics to visit pregnant women in their community has increased as positive impact of the DHFF.

“... all essential requirements for maternal health are available. There is no shortage of drugs, medical reagents, or supplies for maternal health and there is high customer satisfaction with our maternal health services ..." (Community health governing committee members, FGDs)

“... All facility requirements for maternal health services are received on time in our facility and the number of pregnant women who visit our facility increased compared to the previous period before the DHFF ..." (Facility maternity nurse in charge, IDI)

\section{Discussion}

This study evaluated how the DHFF system influenced maternal health-care provision and uptake in Pangani district of Tanzania. The results revealed a positive influence of the system, with strong engagement of community stakeholders during DHFF compared to before this reform. This paper confirms that payment mechanisms like the DHFF are directly linked to the quality of care provided. ${ }^{62}$ When we discuss operational successes and challenges of our findings, we noticed that, during the DHFF intervention, modern equipment for maternal health were procured as compared to the previous interventions and that led to an increasing number of pregnant women attending to primary health facilities for reproductive health services. This means that customers were satisfied with quality of maternal care provided at the health facilities. In the current study, the DHFF set clear operating values that balanced health system capacity with effectiveness, efficiency, and equity that harnessed wider stakeholders' engagement. It also led to shared performance review and increased investment in service delivery processes to address implementation barriers and foster the adoption of best practices. Central government through the ministry of finance is advised to allocate adequate funds to primary health facilities as this will help the facilities to sustain their acquisition of basic needs for provision of quality maternal health services. As a result, the number of women who delivered in health facilities increased significantly by $33.6 \%$, from 1189 to 1589 facility deliveries before and after the DHFF system, respectively.

The management of the DHFF was set in such a way that planning and delivery of maternal health services was participatory and inclusive. Facility health staff and Community representatives played a vital role in the management and execution of DHFF activities, including planning, budgeting, and review of the overall facility performance. ${ }^{63}$ These facilitated interactions between services providers and clients, and provided the primary health facility with opportunities to strengthen community engagement. ${ }^{44,64}$ This approach raised working morale and a sense of ownership among health facility staff, community health governing committee members, and service beneficiaries. $^{34,57}$

Better facility financing improved the overall governance and performance. ${ }^{27} \mathrm{~A}$ similar study in India showed that social accountability mechanisms contributes to improved maternal health outcomes and reduces inequities. ${ }^{65}$ The positive outcomes observed in this study were due to the fact that the DHFF supported health-care workers and community health governing committees to make right decisions based on their needs. It also enabled more regular supportive supervision by the council health management team, and facilitated timely conduct of outreach services that, among other things, stimulated early utilization of maternal health services.

In terms of facility operations, funds were received directly in the bank accounts of respective health facilities. This made it easy to purchase needed maternal health supplies and equipment. Capacity building through training was done to the health facility staff and members of the health governing committee. The DHFF system linked better accounting and reporting systems where financial information from different health facilities simplified report preparation and reduced bureaucracy during procurement processes.

However, breakdown or unavailability of working tools like computers delayed voucher processing. Poor internet connectivity delayed submission of drug requests and their subsequent verification by leaders at the district level. These operational challenges slowed the realization of improved quality care provision and the value expected of the DHFF. A delay in fund disbursement from the government to the facilities led to underperformance. This situation was also seen in a study conducted in Kenya by Opwora et al. ${ }^{44}$ Timeliness is critical to enable facilities to provide services per plans to meet patient expectations and national goals, including providing quality care for women and children.

Our study showed that the direct flow of financial resources to the primary facilities enabled health-care 
workers to meet their budgetary expectations as planned in the facility's annual action plan. This facilitated the procurement of essential requirements for the provision of maternal health services, implying better facility readiness to provide quality maternal health services in terms of antenatal, delivery, and postpartum care. ${ }^{66}$ This also means value for money for all facility requirements purchased and services provided by the facility.

However, other than delivery kits, the DHFF did not bring significant influence to the availability of equipment because it required special permissions from MSD to procure items outside their catalogue. The process of procuring items not on the MSD catalogue could take up to 6 months or more with limited or no certainty of receiving the item. This difficulty was due to the unavailability of a prime vendor for the study region who would be able to supply the items that are unavailable at the MSD. Moreover, to procure items outside MSD required an out-of-stock confirmation letter from MSD, which is difficult to obtain. Again, procuring from local vendors is challenging due to high prices, inconsistent supply, and difficulty in receiving proper and complete documentation such as electronic EFD receipts required for all government purchases. Therefore, this explains the observed inadequate influence of the HDFF on medical equipment at the facility level.

Better availability of essential supplies and equipment encourages maternal health utilization. The attendance of women for antenatal care and delivery in health facilities was recorded higher after, the implementation of the DHFF than before. This was the result of client satisfaction with the services provided at the health facilities. This suggests that service quality informs service uptake. In Rwanda, a similar study showed a positive association between primary healthcare financing and the availability of equipment, drugs, and supply necessary to provide maternal health services. ${ }^{56}$

The direct and explicit government injection of resources for health service delivery is an essential vehicle towards universal health coverage (UHC), improving equity, health outcomes, and financial well-being. ${ }^{67}$ The DHFF is thus propoor as the resources are given to lower health facilities, directly servicing the needy population in rural areas.

Economic growth increases countries' capabilities to increase the share of the health budget and the likelihood to invest in the health sector for quality care. ${ }^{68,69}$ As other countries in eastern and southern Africa, Tanzania has documented a rise in the gross domestic product, and there has been achievements in health system-related investments. ${ }^{70}$ The recently shift of Tanzania from low to middle-income level due to its economic growth, is expected to leap more towards poverty reduction, good governance, and leadership to stimulate action towards provision and utilization of quality health services. ${ }^{70}$ This is necessary for the country to attain UHC and meet the SDGs related to women's and children's health.

\section{Limitations}

A couple of limitations of this study are worthy highlighting. The contribution of the DHFF on maternal health was assessed in one year. This may be too short a period of time to adequately capture its contribution, which, possibly, is why we could not see adequate improvements in equipment. Also, due to financial constraints, this study was conducted in one district only, hence making it difficult to generalize the findings beyond Pangani district. This study was also limited by the lack of adjustment of confounding variables among the women who delivered before and after the DHFF implementation. However, the study was representative of various health facility levels in Tanzania, hence providing a holistic picture of the functionality of the DHFF. Future studies should be conducted with a wider and possibly nationally representative geographical coverage, as well as considering a longer period of observation to comprehensively document the influence of the DHFF on maternal health and other health outcomes in Tanzania.

\section{Conclusions}

The DHFF is functional and improves delivery and uptake of maternal health services in Pangani district in Tanzania. This is due to the increase in the medical supplies, equipment, and reagents necessary for providing maternal health services, thereby facilitating an increase in the number of deliveries at primary health facilities. Furthermore, the DHFF minimizes unnecessary delays in the procurement of items required for service delivery.

Therefore, this study recommends that the DHFF system should be implemented in all health facilities as one of the key health service improvement strategies in Tanzania. Health-care workers should be trained to impart them with the necessary skills and knowledge to ease the implementation of the system. Tailored training for community representatives, the health facility governing committee members to build up their understanding of the system and provide the needed support is required as this will help to allocate funds wisely. Financial technicians to operate the system are important in supporting facility planning and prepare facility financial statement, which complies with DHFF system requirements. Timeliness of 
funds release needs to be prioritized to allow health-care workers to deliver quality care for maternal and other health services. Regular supportive supervision from council health management team to primary health facility is encouraged. This will help to guide facility staff and community health governing members on effective use of the resources for the intended purpose and avoiding unnecessary audit queries. Furthermore, supply of enough computers and strengthening availability of stable internet and electricity to all primary health facilities is recommended as this will increase the impact of the DHFF. The government, policymakers, and other implementing partners need to act upon the DHFF system challenges for the sustainable strengthening of the health system in Tanzania.

\section{Abbreviations}

CHGCM, Community Health Governing Committee Members; CHMT, Council Health Management Team; DHFF, direct health facility financing; DMO, District Medical Officer; FGDs, focus group discussions; HFI, health facility in charge; IDIs, in-depth interviews; P4P, pay for performance; $\mathrm{RCH}$, reproductive and child health; SDGs, sustainable development goals; TBAs, traditional birth attendants; UHC, universal health coverage; MSD, Medical Stores Department.

\section{Data Sharing Statement}

Data were obtained from the Pangani district hospital, Mwera health centre and three dispensaries which were Masaika, Kwakibuyu, and Mkalamo. The datasets used and/or analyzed during the current study are available from the corresponding author on request.

\section{Ethics Approval and Consent to Participate}

Ethical clearance was obtained from the Ifakara Health Institute's Institutional Review Board (IRB) with reference number IHI/IRB/No: 01-2019. Consent to participate in the study was requested from the study participants each of whom signed the consent form before participating in the study. Confidentiality of all the study participants' information was observed and maintained. The Pangani district authority was informed for approval and support. This manuscript was permitted to be published by the National Institute for Medical Research in Tanzania with reference permission number NIMR/HQ/P.12 VOL XXXIII/63.

\section{Acknowledgments}

We acknowledge the help of Pangani district administration, Council Health Management Team, Health facility in charges, Community health governing members, and maternal health beneficiaries from each study facility for their participation in the study. We are grateful for the contribution made by Hajirani Msuya and August Joachim Kuwawenaruwa in this study. Amon Exavery from Pact Tanzania is acknowledged for his technical review and organization of the manuscript.

\section{Author Contributions}

All authors contributed to data analysis, drafting or revising the article, gave final approval of the version to be published, agreed to the submitted journal, and agreed to be accountable for all aspects of the work.

\section{Funding}

Overall, the study was self-funded, except for an ethical clearance fee that the Ifakara Health Institute assisted to cover.

\section{Disclosure}

The authors declare that they have no conflicts of interest for this work.

\section{References}

1. Kuruvilla S, Bustreo F, Kuo T, et al. The Global strategy for women's, children's and adolescents' health (2016-2030): a roadmap based on evidence and country experience. Bull World Health Organ. 2016;94 (5):398-400. doi:10.2471/BLT.16.170431

2. World Health Organization. Trends in Maternal Mortality 2000 to 2017: Estimates by WHO, UNICEF, UNFPA, World Bank Group and the United Nations Population Division. WHO, UNICEF, UNFPA, World Bank Group and the United Nations Population Division; 2017. 104.

3. Presterl E, Diab-El Schahawi M, Lusignani LS, Paula H, Reilly JS. Reprocessing: cleansing, disinfection, sterilization. In: Basic Microbiology and Infection Control for Midwives. Springer; 2019:35-49.

4. Thapa P, Bangura AH, Nirola I, et al. The power of peers: an effectiveness evaluation of a cluster-controlled trial of group antenatal care in rural Nepal. Reprod Health. 2019;16(1):1-14. doi:10.1186/s12978019-0820-8

5. Bauserman M, Lokangaka A, Thorsten V, et al. Risk factors for maternal death and trends in maternal mortality in low- and middle-income countries: a prospective longitudinal cohort analysis. Reprod Health. 2015;12(2):1-9. doi:10.1186/1742-4755-12-S2-S5

6. Nyamtema A, Mwakatundu N, Dominico S, et al. Introducing eHealth strategies to enhance maternal and perinatal health care in rural Tanzania. Matern Health Neonatol Perinatol. 2017;3(1):3. doi:10.1186/s40748-017-0042-4

7. Kassebaum NJ, Bertozzi-Villa A, Coggeshall MS, et al. Global, regional, and national levels and causes of maternal mortality during 1990 2013: a systematic analysis for the Global Burden of Disease Study 2013. Lancet. 2014;384(9947):980-1004. doi:10.1016/S01406736(14)60696-6 
8. Arsenault C, Jordan K, Lee D, et al. Equity in antenatal care quality: an analysis of 91 national household surveys. Lancet Glob Health. 2018;6(11):e1186-e1195. doi:10.1016/S2214-109X(18)30389-9

9. Hanson C, Gabrysch S, Mbaruku G, et al. Access to maternal health services: geographical inequalities, United Republic of Tanzania. 2017;95:810-820. Bull World Health Organ. doi:10.2471/ BLT.17.194126

10. Tunçalp Ö, Pena-Rosas J, Lawrie T, et al. WHO recommendations on antenatal care for a positive pregnancy experience-going beyond survival. BJOG. 2017;124(6):860-862. doi:10.1111/1471-0528.14599

11. Al-beity FA, Pembe AB. "We do what we can do to save a woman" health workers' perceptions of health facility readiness for management of postpartum haemorrhage. Glob Health Action. 2020;13 (1):1-12.

12. Mkoka DA, Mahiti GR, Kiwara A, Mwangu M, Goicolea I, Hurtig A-K. "Once the government employs you, it forgets you": health workers' and managers' perspectives on factors influencing working conditions for provision of maternal health care services in a rural district of Tanzania. Hum Resour Health. 2015;13(1):77. doi:10.1186/s12960-015-0076-5

13. Macdonald D, Aston M, Murphy GT, et al. Providing postpartum care with limited resources: experiences of nurse-midwives and obstetricians in urban Tanzania. Women Birth. 2018;32:e391-e398 doi:10.1016/j.wombi.2018.07.016

14. Thomson S, Foubister T, Mossialos E. Financing Health Care in the European Union. WHO; 2009.

15. Saronga HP, Duysburgh E, Massawe S, et al. Efficiency of antenatal care and childbirth services in selected primary health care facilities in rural Tanzania: a cross-sectional study. BMC Health Serv Res. 2014;14:1-11. doi:10.1186/1472-6963-14-96

16. Shabani J, Todd G, Nswilla A. Maternal Mortality in Urban and Rural Tanzania Social Determinants and Health System Efficiency. International Growth Center; 2018.

17. Kabia E, Mbau R, Oyando R, Oduor C, Bigogo G, Khagayi S. "We are called the et cetera": experiences of the poor with health financing reforms that target them in Kenya. Int J Equity Health. 2019;2:1-14.

18. Id VV, Ahmed S, Adams AM. Factors enabling comprehensive maternal health services in the benefits package of emerging financing schemes: a cross- sectional analysis from 1990 to 2014. PLoS One. 2018;13:1-15.

19. Xu K, Evans DB, Carrin G, Aguilar-Rivera AM, Musgrove P, Evans T. Protecting households from catastrophic health spending. Health Aff. 2007;26(4):972-983. doi:10.1377/hlthaff.26.4.972

20. Kutzin J. Practice health financing for universal coverage and health system performance: concepts and implications for policy. Bull World Health Organ. 2013;91:602-611. doi:10.2471/BLT.12.113985

21. Lozano R, Fullman N, Abate D, et al. Measuring progress from 1990 to 2017 and projecting attainment to 2030 of the health-related Sustainable Development Goals for 195 countries and territories: a systematic analysis for the Global Burden of Disease Study 2017. Lancet. 2018;392(10159):2091-2138.

22. Mills A, Ataguba JE, Akazili J, et al. Equity in financing and use of health care in Ghana, South Africa, and Tanzania: implications for paths to universal coverage. Lancet. 2012;380(9837):126-133. doi:10.1016/S0140-6736(12)60357-2

23. Mtei G, Mulligan J, Palmer N, Kamuzora P, Ally M, Mills A. An assessment of the health financing system in Tanzania: implications for equity and social health insurance. 2007.

24. Barugahare J, Lie RK. Obligations of low income countries in ensuring equity in global health financing. BMC Med Ethics. 2015;16 (1):1-11. doi:10.1186/s12910-015-0055-3

25. Songstad NG, Rekdal OB, Massay DA, Blystad A. Perceived unfairness in working conditions: the case of public health services in Tanzania. BMC Health Serv Res. 2011;11(1):34. doi:10.1186/14726963-11-34
26. Frumence G, Nyamhanga T, Mwangu M, Hurtig AK. Challenges to the implementation of health sector decentralization in Tanzania: experiences from kongwa district council. Glob Health Action. 2013;6(1). 20983. doi:10.3402/gha.v6i0.20983

27. USAID. Health Financing Profile - Tanzania. Health Policy Project; 2016:1-2.

28. Frumence G, Nyamhanga T, Mwangu M, Hurtig AK. Challenges to the implementation of health sector decentralization in Tanzania: experiences from Kongwa district council. Glob Health Action. 2013;6:9716.

29. Borghi J, Mayumana I, Mashasi I, et al. Protocol for the evaluation of a pay for performance programme in Pwani region in Tanzania: a controlled before and after study. Implement Sci. 2013;8(1):1-12. doi:10.1186/1748-5908-8-80

30. Chimhutu V, Tjomsland M, Mrisho M. Experiences of care in the context of payment for performance (P4P) in Tanzania. Glob Health. 2019;9:1-13.

31. Songstad NG, Lindkvist I, Moland KM, Chimhutu V, Blystad A. Assessing performance enhancing tools: experiences with the open performance review and appraisal system (OPRAS) and expectations towards payment for performance (P4P) in the public health sector in Tanzania. Global Health. 2012;8:1-13. doi:10.1186/17448603-8-1

32. Chimhutu V, Songstad NG, Tjomsland M, Mrisho M, Moland KM. The inescapable question of fairness in Pay- for-performance bonus distribution: a qualitative study of health workers ' experiences in Tanzania. Glob Health 2016;15:1-12.

33. Janus $\mathrm{H}$, Keijzer N. Big results now? Emerging lessons from results-based aid in Tanzania. SSRN Electron J. 2015. doi:10.2139/ssrn.2673227

34. Borghi J, Little R, Binyaruka P, Patouillard E, Kuwawenaruwa A. In Tanzania, the many costs of pay-for-performance leave open to debate whether the strategy is cost-effective. Health Aff. 2015;34 (3):406-414. doi:10.1377/hlthaff.2014.0608

35. McIntyre D, Gilson L, Mutyambizi V. Promoting equitable health care financing in the African context: current challenges and future prospects. 2005.

36. Kapologwe NA, Kibusi SM, Borghi J, Gwajima DO, Kalolo A. Assessing health system responsiveness in primary health care facilities in Tanzania. BMC Health Serv Res. 2020;20(1):104. doi:10.1186/s12913-020-4961-9

37. Witter S, Toonen J, Meessen B, Kagubare J, Fritsche G, Vaughan K. Performance-based financing as a health system reform: mapping the key dimensions for monitoring and evaluation. BMC Health Services Res. 2013;13:1-10.

38. WHO. Health System Responsiveness. WHO; 2010.

39. Piatti-Fünfkirchen M, Schneider P. From stumbling block to enabler: the role of public financial management in health service delivery in Tanzania and Zambia. Health Syst Reform. 2018;4:336-345. doi:10.1080/23288604.2018.1513266

40. Zeng W, Shepard DS, De DIeu Rusatira J, Blaakman AP, Nsitou BM. Evaluation of results-based financing in the Republic of the Congo: a comparison group pre-post study. Health Policy Plan. 2018;33 (3):392-400. doi:10.1093/heapol/czx195

41. Mwakatumbula BH. The Implementation of Direct Health Facility Financing (DHFF): prospects and challenges. 2021.

42. Baseline Report. Prospects for sustainable health financing in Tanzania; 2015. Available from: https://www.healthpolicyproject. com/pubs/788_ProspectsforSustainableHealthFinanciFINAL.pdf. Accessed November 17, 2021.

43. Alliance for Health Policy and Systems Research; Bill \& Melinda Gates Foundation; WHO. Report of the Expert Consultation on Primary Care Systems Profiles \& Performance (PRIMASYS); 2015. Available from: https:/www.who.int/alliance-hpsr/projects/ PRIMASYS_Expert_Consultation_Final_Report.pdf?ua=1. Accessed December 1, 2021. 
44. Opwora A, Kabare M, Molyneux S, Goodman C. The implementation and effects of direct facility funding in Kenya's Health Centres and Dispensaries. 2009:1-28.

45. Opwora A, Kabare M, Molyneux S, Goodman C. Direct facility funding as a response to user fee reduction: implementation and perceived impact among Kenyan health centres and dispensaries. Health Policy Plan. 2010;25(5):406-418. doi:10.1093/heapol/czq009

46. Waweru E, Opwora A, Toda M, et al. Are Health Facility Management Committees in Kenya ready to implement financial management tasks Findings from a nationally representative survey. BMC Health Serv Res. 2013;13(1):1-14. doi:10.1186/1472-6963-13-404

47. Kapologwe NA. Direct Health Facility Financing (DHFF) in Tanzania. 2018.

48. NIMR. Evidence-Informed Policy Making in the United Republic of Tanzania: Setting REACH-Policy Initiative Priorities for EvidenceInformed Policy Making in the United Republic of Tanzania: Setting REACH-Policy Initiative Priorities for 2008-2010. Report; 2010.

49. Kapologwe NA, Meara JG, Kengia JT, Sonda Y, Gwajima D. Development and upgrading of public primary healthcare facilities with essential surgical services infrastructure: a strategy towards achieving universal health coverage in Tanzania. BMC Health SRes. 2020;2:1-14.

50. Boex J, Fuller L, Malik A. Decentralized local health services in Tanzania. Urban Instuition; 2015. Available from: https://www.urban. org/sites/default/files/publication/51206/2000215-DecentralizedLocal-Health-Services-in-Tanzania.pdf. Accessed November 17, 2021.

51. Boex J, Fuller L, Malik A. Decentralized Local Health Services in Tanzania. Urban Instituion; 2015.

52. Gilson L, Sen PD, Mohammed S, Mujinja P. Local government decentralization and the health sector in Tanzania. Health Policy Plan. 1994;9:14. doi:10.1093/heapol/9.1.14

53. Piatti-Fünfkirchen M, Ally M. Tanzania health sector public expenditure review 2020. 2020.

54. World Health Organization. Primary Health Care Systems (Primasys). World Health Organization; 2017.

55. Wiedenmayer K, Mbwasi R, Mfuko W, et al. Jazia prime vendor system- a public-private partnership to improve medicine availability in Tanzania: from pilot to scale. J Pharm Policy Pract. 2019;4:1-10.

56. Tong A, Sainsbury P, Craig J. Consolidated criteria for reporting qualitative research (COREQ): a 32-item checklist for interviews and focus groups. Int J Qual Health Care. 2007;19(6):349-357. doi:10.1093/intqhe/mzm042

57. Bonde AH, Stjernqvist NW, Sabinsky MS, Maindal HT. Process evaluation of implementation fidelity in a Danish health-promoting school intervention. BMC Public Health. 2018;18(1):1-10. doi:10.1186/s12889-018-6289-5
58. Carroll C, Patterson M, Wood S, Booth A, Rick J, Balain S. A conceptual framework for implementation fidelity. Implement Sci. 2007;2(1):1-9. doi:10.1186/1748-5908-2-40

59. Breitenstein SM, Gross D, Garvey CA, Hill C, Fogg L, Resnick B. Implementation fidelity in community-based interventions. Res Nurs Health. 2010;33(2):164-173. doi:10.1002/nur.20373

60. Cissell WB. Process evaluation for public health interventions and research. Health Educ Res. 2004;19(6):739. doi:10.1093/her/cyg084

61. Ranson MK, Hanson K, Oliveira-Cruz V, Mills A. Constraints to expanding access to health interventions: an empirical analysis and country typology. J Int Dev J Dev Stud Assoc. 2003;15(1):15-39.

62. McLoughlin V, Leatherman S. Quality or financing: what drives design of the health care system? Qual Saf Health Care. 2003;12 (2):136-142. doi:10.1136/qhc.12.2.136

63. Tiruneh GT, Zemichael NF, Betemariam WA, Karim AM. Effectiveness of participatory community solutions strategy on improving household and provider health care behaviors and practices: a mixed-method evaluation. Hurley EA, editor. PLoS One. 2020;15(2):e0228137. doi:10.1371/journal.pone.0228137

64. Macha J, Borghi J. Health facility committees: are they working? IHI. 2011;7(7):1-4.

65. Hamal M, De Cock Buning T, De Brouwere V, Bardají A, Dieleman M. How does social accountability contribute to better maternal health outcomes? A qualitative study on perceived changes with government and civil society actors in Gujarat, India. BMC Health Serv Res. 2018;18(1):1-15. doi:10.1186/s12913-018-3453-7

66. Bintabara D, Ernest A, Mpondo B. Health facility service availability and readiness to provide basic emergency obstetric and newborn care in a low-resource setting: evidence from a Tanzania National Survey. BMJ Open. 2019;9(2):1-10. doi:10.1136/bmjopen-2017-020608

67. Wang H, Juma MA, Rosemberg N, Ulisubisya MM. Progressive pathway to universal health coverage in Tanzania: a call for preferential resource allocation targeting the poor. Health Syst Reform. 2018;4(4):279-283. doi:10.1080/23288604.2018.1513268

68. Piatti-fünfkirchen M, Lindelow M, Yoo K, Piatti-fünfkirchen M, Lindelow M, Yoo K. What are governments spending on health in East and Southern Africa? Health Syst Reform. 2018;4(4):284-299. doi:10.1080/23288604.2018.1510287

69. Moucheraud C, Owen H, Singh NS, et al. Countdown to 2015 country case studies: what have we learned about processes and progress towards MDGs 4 and 5? BMC Public Health. 2016;16 (Suppl 2). doi:10.1186/s12889-016-3401-6

70. Moyo M, Simson R, Jacob A, De MF. Attaining Middle Income Status - Tanzania: Growth and Structural Transformation Required to Reach Middle Income Status by 2025. International Growth Centre, London School of Economics and Political Science; 2012:44.

\section{Publish your work in this journal}

The International Journal of Women's Health is an international, peerreviewed open-access journal publishing original research, reports, editorials, reviews and commentaries on all aspects of women's healthcare including gynecology, obstetrics, and breast cancer. The manuscript management system is completely online and includes a very quick and fair peer-review system, which is all easy to use. Visit http://www.dovepress.com/testimonials.php to read real quotes from published authors. 